\title{
027 The Last Ten Years of Traditional Craftsmanship in Miaoxia Village
}

Kuo Jze Yi

$118-129$
This article reflects on the disappearing carpentry tradition in a rural village called Miaoxia in Sichuan Province China. Since 2015, villagers, social workers, architects, and university scholars have been collaborating to look for alternative development possibilities in Miaoxia Village. The idea of using the local carpentry tradition has been one of the key focusses in the process. Since the Chinese Economic Reform in 1978, the influence of urbanisation and market economy in China has led the Chinese government to rethink the value of rural customs and traditions. While the country has been encouraging progressive economic development, local making culture and development have subsequently been under threat. The collaborations between social workers and design professions in Miaoxia tested small-scale architecture interventions and educational workshops. These experiments have started to record and test out different ways to save carpentry traditions from extinction. This \#craft \#tradition \#community development \#workshop article outlines this process in Miaoxia and asks for new ideas to re-utilise this traditional making. 


\section{Introduction}

The development of rural settlements in Sichuan province of the People's Republic of China (PRC) was based on the residents' livelihood opportunities and local resources. Throughout the centuries, rural architecture formed and transformed according to necessities, traditions and the ideas of the local people. The old quarter of Miaoxia village in Yaan City, Sichuan province, China, is a cluster of thirty to forty remaining timber houses around the village square. These timber houses were built with a traditional flexible timber frame system called chuan dou, which can be modified and adapted to sit on different topography and shapes of land. The carpenters connect structural elements with joinery and no nails, allowing them to be easily taken apart and reconnected. This flexible building system is not only resistant to severe earthquakes, and adaptable to many types of spatial arrangement, but it has also allowed many villagers to transport some houses from other locations to their current locations in Miaoxia. Unfortunately, the demand for this building type is declining, because of the mass production and large-scale building industry of concrete frame structures, and also the PRC's environmental policies regarding the preservation of trees. At the same time, researchers feel the need to preserve traditional culture and wisdom, while finding alternative and appropriate development directions for the future. Facing this dilemma of possible cultural and traditional extinction induced by progressive development, how can architectural researchers and makers explore new possibilities and new ideas?

\section{Experiment 1: Small-Scale Intervention}

Since November 2014, Associate Professor Peter Hasdell of The Hong Kong Polytechnic University
School of Design has collaborated with Associate Professor Ku Hok Bun of the Department of Applied Social Sciences, to research alternative bottom-up development strategies for Miaoxia Village, where social workers from the Lugeng Center for Advancement of Rural Urban Sustainability were stationed for three years. The first collaboration of social workers and architects resulted in the completion of a community kitchen building that hosted community events and visitors, thus generating an income for villagers. More recently a community guesthouse building was completed in January 2018. These projects together continue to test and develop the bottom-up community development approach framed by the project team's research.

One of the key design decisions in both building projects was to utilise and reinvent the traditional timber frame system to construct both structures. During the process local shifu, or "old masters," were commissioned to participate in both the design and construction stages. For the community kitchen, the structural columns plan is exactly the same as the traditional grids set up for houses and other buildings, but the height of each column was adjusted to form a lifted roof structure that opens to the square. To accomplish this design intent, masters only needed to know each column height and modified roof connection details. For the second structure, the community guesthouse, the building plan is angled in the central space to form a diagonal column line, to provoke a new type of interior space and building mass. Similar to the first project, the masters only needed a floor plan indicating column positions and each column's height. Thereafter, step by step, they could resolve different angled joinery and connections. Although this process of utilizing and reinventing traditional building methods seems nostalgic and demanding, it is an arguably faster and cheaper alternative to having these buildings constructed in concrete frames by a regional contracting company. Since the building scale is rather small, and 
because the villagers can collectively participate in some of the low-skilled and labour-intensive building processes, the projects were therefore economically viable. Through their participatory model and adaptation of local craftsmanship, these projects focus on the social and cultural aspects rather than merely completing a building. From this example, one can foresee opportunities to continue developing traditional building systems if the development remains focused on the small scale, aiming for direct engagement with small communities.

\section{Experiment 2: Rural Craft Workshop}

The timber frame system and carpentry tradition in Miaoxia village attracted design students from three major institutions: the Hong Kong Design Institute, The Hong Kong Polytechnic University, and Shenzhen University. Between July 2015 and July 2016 researchers and local craftspeople hosted three carpentry workshops in the village. The researchers also hosted one building workshop based on the same timber structural system at The Hong Kong Polytechnic University during the 2015 Maker Faire event on campus (Fig. 12). Since carpentry tools and timber logs are exotic items to students in Hong Kong, many students expressed curiosity and the will to work with them during the workshops. The workshops took place over the course of ten to fourteen days.

They tested the potentials of traditional craft in relation to modern generations, and also tested whether traditional craft workshops could become a source of income for Miaoxia village. All four workshops generated intriguing outcomes, but whether this method could become an economically sustainable programme requires more commitment from interested locals. Currently, these craft workshops can only be held seasonally for a maximum of thirty of university students at a time. To be economically sustainable, craft workshops need to be frequent, and the model may benefit from integrating into design education programmes. Historically, students reported gaining practical skills and imaginative ideas from the masters, particularly from a workshop held in July 2016 in Miaoxia village. Perhaps if university curriculums work with villages to pilot one-to-one studies, it could create meaningful and sustainable opportunities for both academia and the village.

The Miaoxia workshop in July 2016 was called "Re-interpreting Rural Furniture." Students were asked to:

1. Discover local stories in Miaoxia;

2. Practise carpentry or bamboo weaving techniques with local masters;

3. Create furniture that re-interprets local stories; and

4. Document traditional craft and local stories.

Eighteen architecture students from the Hong Kong Design Institute and Shenzhen University applied to join the workshop. They were divided into five groups, practising carpentry with masters while discovering local stories. This resulted in the students proposing and prototyping five furniture projects narrating different local stories. The workshop documented these local stories and traditional crafts, engaged old masters and villagers, tested possibilities to connect design students to the rural traditions, and created inventive design ideas based on local resources. This experiment portrays opportunities to creatively preserve and extend traditional wisdom, if researchers align design education with the rural villages. Below are the brief outlines of the five project outcomes.

Group One students followed a family of grandparents and two grandchildren to observe how they live together. This extended family structure 
is a common social dynamic in China, due to the increasing number of parents becoming migrant workers and consequently having to leave children behind with grandparents. This group of students discovered that benches in the village have nearly uniform heights, which do not permit the small children to sit at eye level with the taller grandparents. The students proposed to build a bench that was higher on one side for the children and lower on the other side for the grandparents, thus allowing both children and grandparents to be on the same height level. Due to students' inexperience, the project prototype was built too small, resulting in the grandfather and the grandchild sitting back to back (Fig. 5).

Group Two students followed six children in the village, and understood through interviews that the children wanted a collective playground space. The complicated land ownership structures in the rural area, however, did not offer conditions for building a playground. Eventually, the group of students proposed to build a portable slide that can be carried around by children, to avoid occupying a fixed location, and to help solidify their friendship through carrying the structure around together (Fig. 6).

Group Three students were fascinated by occasional community-gathering events, such as meal gatherings or outdoor film viewings. Villagers would bring chairs from home or benches from neighbouring houses to join the event. However, this region is known for its unpredictable weather and oftentimes rain would bring the gatherings to an abrupt end, thus forcing the villagers to return home. The group of students built a table that can be flipped around to form a rain shelter, so community gatherings would not be abruptly halted by unpredictable weather (Fig. 7).

Group Four students found that meandering village passages are confusing for visitors. Therefore, students proposed designs for a wayfinding system.
Eventually the students built a signpost, modified from a traditional timber frame. The organic fabric of the settlement led them to a diagonal intersecting structure, which indicates different directions while simultaneously forming a selfsupporting bench for passers-by to stop and rest.

Group Five students were fascinated by how villagers hammered nails into walls of traditional timber structures to hang bags for storage. This storage method seemed practical, but haphazard and damaging to the wooden houses. Therefore, these students proposed a new joinery that clamps and loops around the timber structure to avoid damaging the old building whilst simultaneously providing storage space.

\section{Craft as a Form of Ideas}

Miaoxia village was famed for its carpenters. The master carpenters have a particular attitude, and are respected among their communities. They keep a straight and sturdy posture and speak calmly and confidently to communicate ideas. They are in charge of the ink (墨 斗 or mo dou, in traditional Chinese and pinyin), a tool that contains string and ink to mark on the timber logs, to determine the measurement and alignment of the whole structure. Junior carpenters shape the timber logs and chisel joinery. Once the frame is constructed flat on the ground, villagers gather to lift up the structural frames, and the carpenters climb up the frames to connect them, one by one. Once the structure is erected, the carpenters settle the roof rafting and the villagers stand in a line to pass along the roof tiles up to the top of the structure to lay the final layer of the roof. This is how the villagers have built their houses collectively throughout the centuries.

The tradition of building houses is not only about construction; it also involves a ritual ceremony, customs, beliefs and mysteries. For example, 
the villagers stated that the highest beam of the traditional house structure must be felled on the day the builders raise the structural frame. Furthermore, the tree trunk must be of a particular tree, belonging to another villager. This tree trunk is discretely cut in the early morning without the owner being informed and money is left at the root of the tree trunk to be collected upon the owner's return. Unfortunately, this sophisticated form of communal relationship is also facing extinction.

Miaoxia carpentry tradition was a form of cultural practice, community bonding ritual, economic exchange, and self-discipline. It is one of the unique local traditions that represented the community. Nowadays, the youngest master carpenters are approximately sixty years old. In a decade, this sophisticated culture will be lost forever. The community kitchen project, guesthouse project, and carpentry workshops can be seen as experiments; the project explores and documents the traditions to initiate new opportunities. In this process, Miaoxia carpentry has transformed from skills into design ideas and educational ideas. As research to redefine crafting, it positions making processes as design ideas. As the villagers' ages and social contexts change, it is highly unrealistic and unproductively conservative to wish to maintain craft traditions exactly as they are. By adapting traditional ideas, not merely repeating tedious craftsmanship outcomes, this transformation of practice might offer new imaginative opportunities. Researchers, students, and villagers together can then continue to relate ideas to our history, and extend it to create both familiar and exciting new conditions in architecture.

\section{Acknowledgements}

\author{
Miaoxia Villagers \\ Carpentry Masters \\ Yang Shi Kang, Luo Rong Bin \\ Lugeng Center for the Advancement of \\ Rural Urban Sustainability
}

Qi Hua Dong, Yang Li Zhen, Jing Yeng Shi, Qiang

Zhao Xing, Ren Hau

The Hong Kong Polytechnic University

Department of Applied Social Sciences (APSS)

Dr. Ku Hok Bun

In-situ Project

(Design Research Leadership Team)

Peter Hasdell, Kuo Jze Yi, Tan Min, Clarence Ku

Hong Kong Design Institute

Student Collaborators

Chi Ho Chung, Randolph Yik Sun Lai, Theodora Tin Wai Li, Wing Chun Cheng, Wallace Hon Fung Wu, Tak Kim Chu, Stefan Kai Hin Chui, Sai Yiu Yu, Wai Kit Chu, Kelvin Chun Yin Cheung, Ethan Chi To Shum, Hau En Tsai, Suet Ngo Yan, Marco Leung Yin Sing, Horace Chi Ho Yeung, Luis Shing, Sharon Tsoi Yuk Ying, Brian Kai Ho Au, Teresa Tin, Eason Kit Yeung, Chuk Ming Wong, Shing Lap Sy

Shenzhen University Student Collaborators

Zhang Hui Xing, Hong Bi Sheng, Li Shi Zhao, Lin Qi Qing, Chen Xin, Zhu Lan, Tan Chu Jun, Xie Jing Yi, Deng Yuan, Deng Zhi Jian, Weng Ce Kai. 

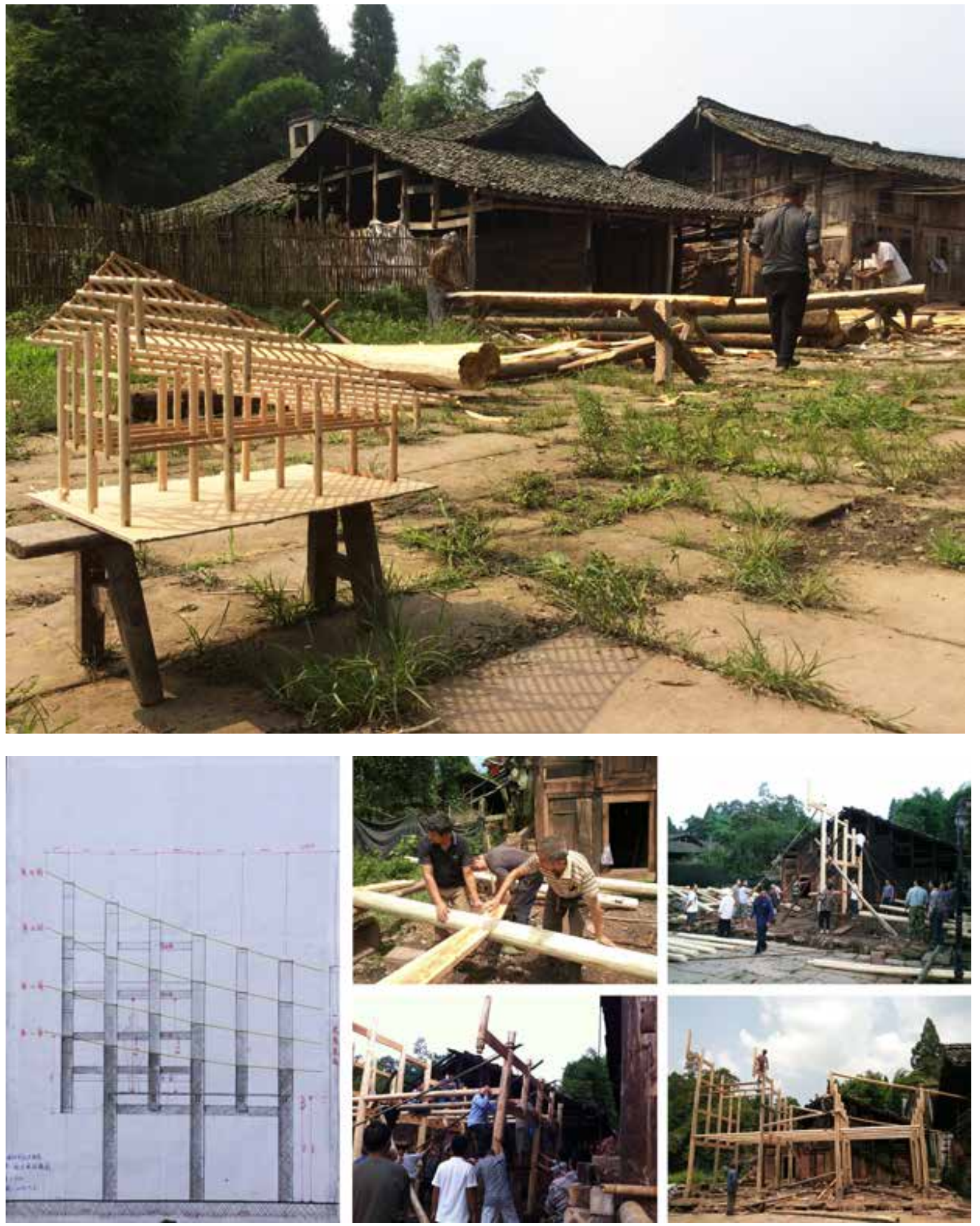

Figure 1 (top): Miaoxia Community Kitchen 1:25 Model, near the site of the kitchen structure. Source: author.
Figure 2a-e (bottom): Chuan Dou construction sequence, Miaoxia community kitchen under construction in July 2015. Source: author. 

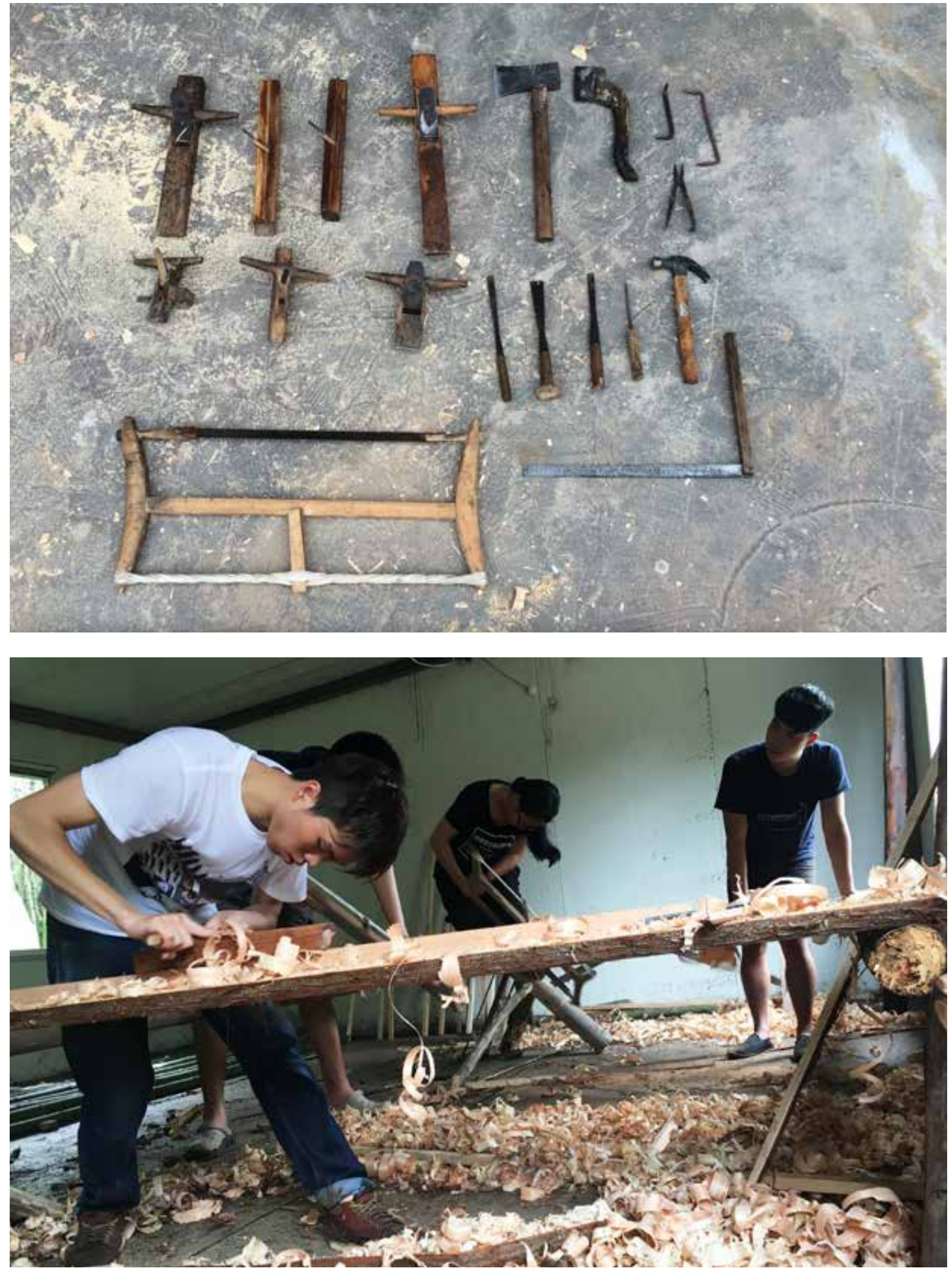

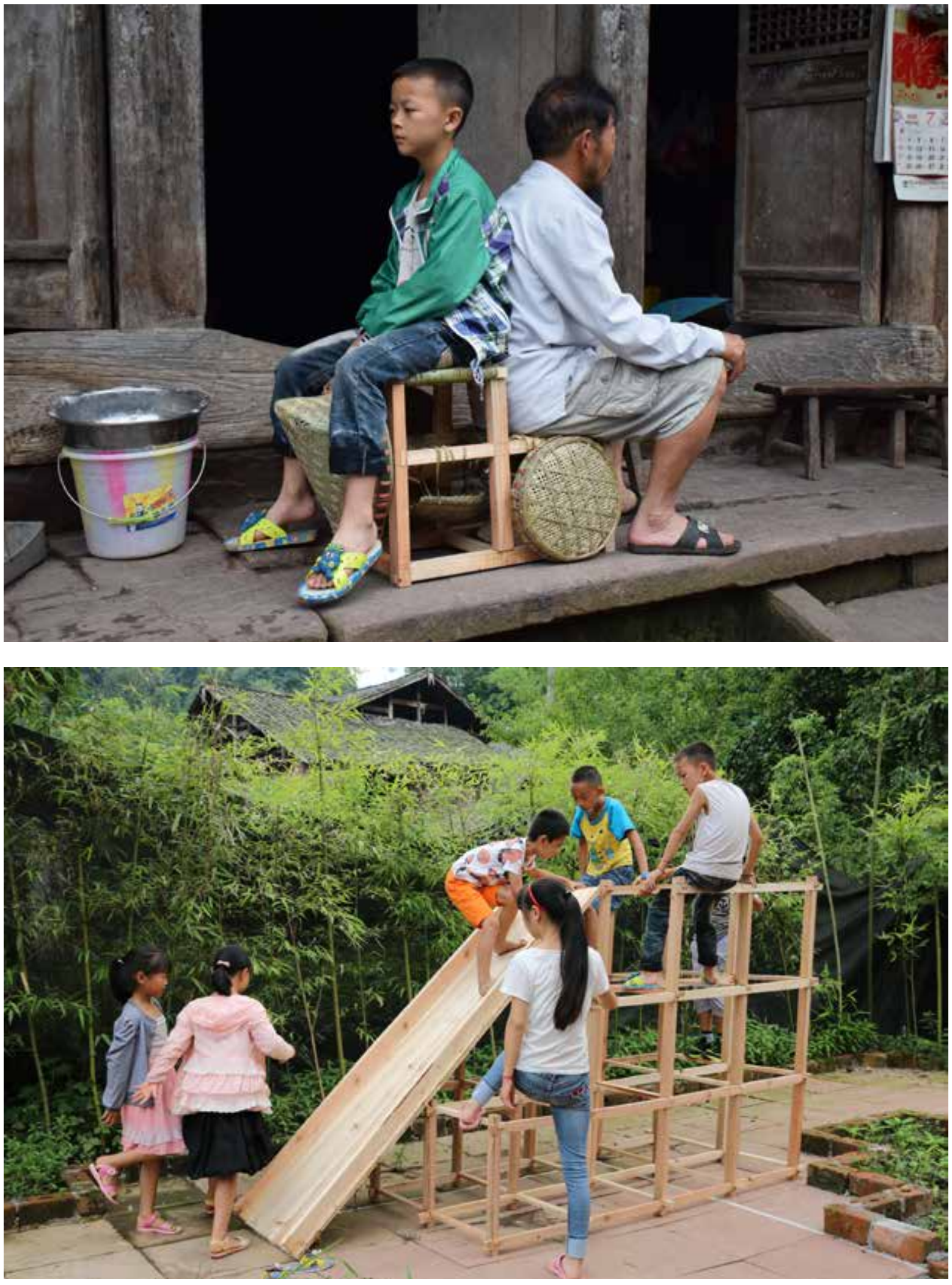



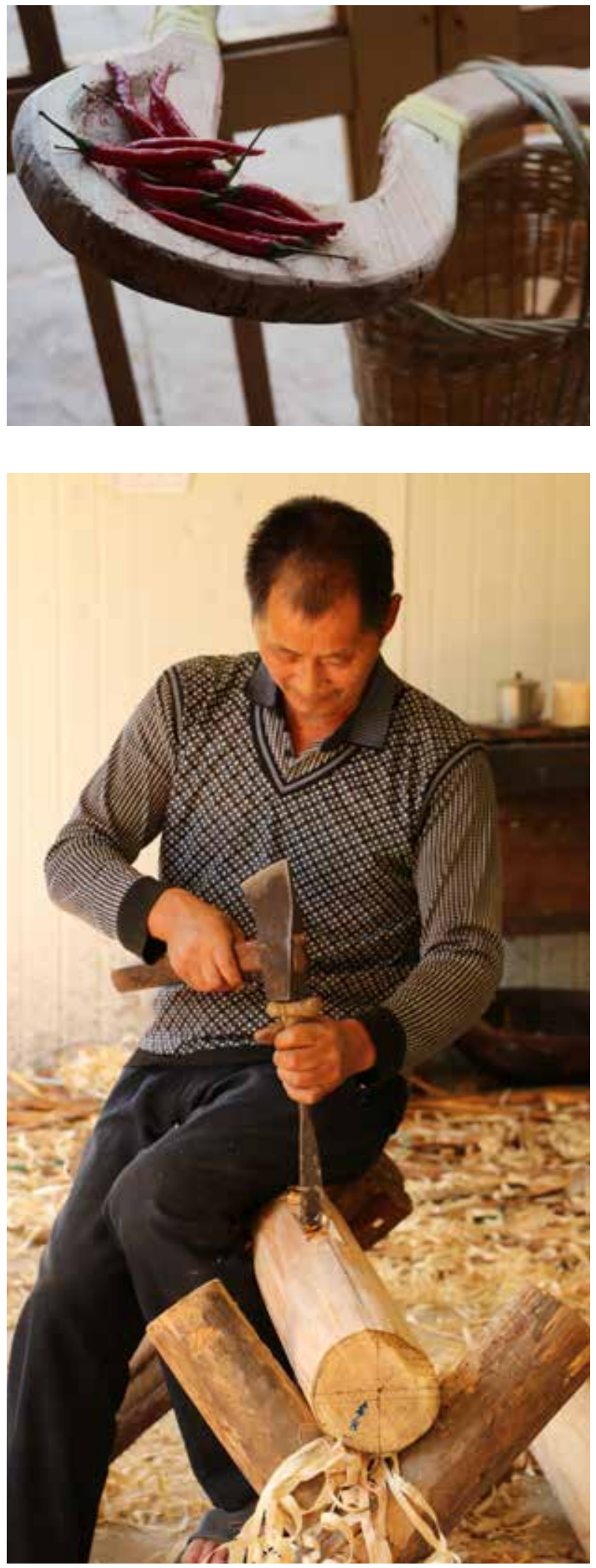

Figure 3-4 (page 124): Carpentry tools in Miaoxia Village. Miaoxia craft workshop in July 2016. Source: author.

Figure 5 (top, page 125): Student Craft Workshop Group One. Bench of Different Height designed by Chi Ho Chung, Zhang Hui Xing and Li Shi Zhao. Source: author.

Figure 6 (bottom, page 125): Student Craft Workshop Group Two. Portable Slide designed by Theodora Tin Wai Li, Luis Shing, Chen Xin, Deng Zhi Jian. Source: author.

Figure $\mathbf{7}$ (top, opposite page): Student Craft Workshop Group Three. Rain Proof Furniture design by Marco Yin Sing Leung, Suet Ngo Yan, Xie Jing Yi, Hong Bi Sheng. Source: author.

Figure 8 (bottom, opposite page): Student Craft Workshop Group Four. Diagonal Signpost design by Horace Chi Ho Yeung, Sharon Yuk Ying Tsoi, Deng Yuan, Weng Ce Kai. Source: author.

Figure 9 (top): Student Craft Workshop Group Five. Twisted Interior Furniture design by Lin Qi Qing, Zhu Lan, Tan Chu Jun. Source: author.

Figure 10 (bottom): Master Carpenter Posture. Source: author.

Figure 11 (top, page 128): Erecting the structure for the Miaoxia Community Kitchen in July 2015. Source: author.

Figure 12 (bottom, page 128): Visitors to the 2015 Maker Faire at The Hong Kong Polytechnic University learned carpentry techniques from the students who participated in the 2015 Miaoxia workshop. Source: author. 

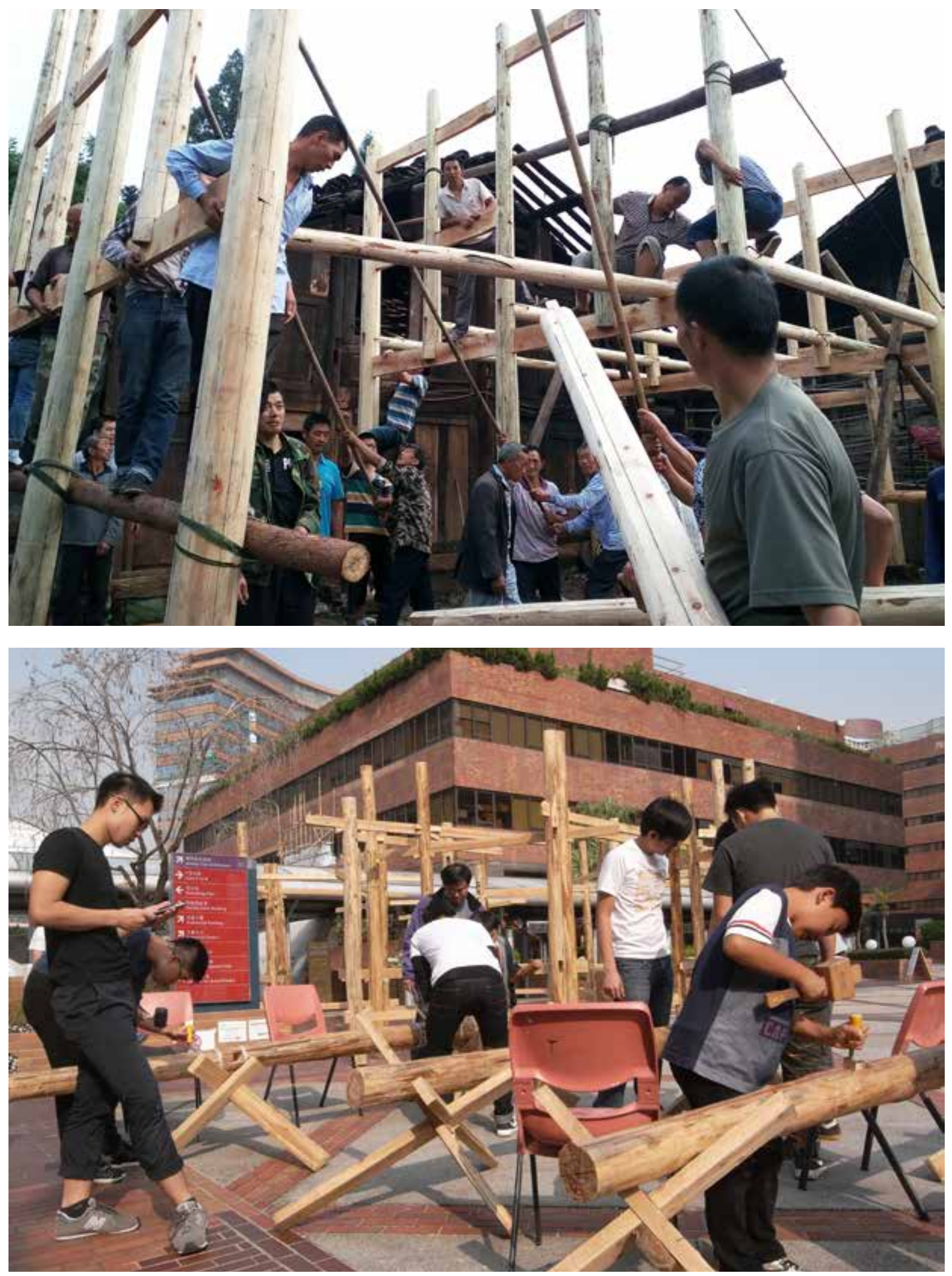


\section{Bio}

Kuo Jze $\mathbf{Y i}$ is an architect and researcher. His research focuses on participatory rural community development and sustainable rural building techniques. From 2015 until currently, Kuo has participated in eight community projects in rural China and completed ten community building designs that adapted traditional building techniques of timber frame, stone construction, cave construction, bamboo structure and building with local resources. He has also organised fifteen workshops in rural China, guiding 250 volunteers from different schools to document and explore traditional building culture. Kuo is currently the assistant professor of the Shenzhen University School of Architecture and Urban Planning and the visiting lecturer in The Hong Kong Polytechnic University School of Design. 